\title{
Improvement of Adequate Digoxin Dosage: An Application of Machine Learning Approach
}

\author{
Ya-Han Hu $\mathbb{D}^{1,2}$ Chun-Tien Tai, ${ }^{1,3}$ Chih-Fong Tsai $\mathbb{D}^{4},{ }^{4}$ and Min-Wei Huang ${ }^{5,6}$ \\ ${ }^{1}$ Department of Information Management and Institute of Healthcare Information Management, \\ National Chung Cheng University, Chiayi, Taiwan \\ ${ }^{2}$ Center for Innovative Research on Aging Society (CIRAS), National Chung Cheng University, Chiayi, Taiwan \\ ${ }^{3}$ Chiayi Chang Gung Memorial Hospital, Chiayi, Taiwan \\ ${ }^{4}$ Department of Information Management, National Central University, Taoyuan, Taiwan \\ ${ }^{5}$ School of Medicine, China Medical University, Taichung, Taiwan \\ ${ }^{6}$ Department of Psychiatry, Chiayi Branch, Taichung Veterans General Hospital, Chiayi, Taiwan \\ Correspondence should be addressed to Min-Wei Huang; mingwei.huang16@gmail.com
}

Received 1 March 2018; Revised 15 June 2018; Accepted 22 July 2018; Published 19 August 2018

Academic Editor: Vincenzo Positano

Copyright (c) 2018 Ya-Han Hu et al. This is an open access article distributed under the Creative Commons Attribution License, which permits unrestricted use, distribution, and reproduction in any medium, provided the original work is properly cited.

\begin{abstract}
Digoxin is a high-alert medication because of its narrow therapeutic range and high drug-to-drug interactions (DDIs). Approximately $50 \%$ of digoxin toxicity cases are preventable, which motivated us to improve the treatment outcomes of digoxin. The objective of this study is to apply machine learning techniques to predict the appropriateness of initial digoxin dosage. A total of 307 inpatients who had their conditions treated with digoxin between 2004 and 2013 at a medical center in Taiwan were collected in the study. Ten independent variables, including demographic information, laboratory data, and whether the patients had CHF were also noted. A patient with serum digoxin concentration being controlled at $0.5-0.9 \mathrm{ng} / \mathrm{mL}$ after his/her initial digoxin dosage was defined as having an appropriate use of digoxin; otherwise, a patient was defined as having an inappropriate use of digoxin. Weka 3.7.3, an open source machine learning software, was adopted to develop prediction models. Six machine learning techniques were considered, including decision tree $(\mathrm{C} 4.5), k$-nearest neighbors $(\mathrm{kNN})$, classification and regression tree (CART), randomForest (RF), multilayer perceptron (MLP), and logistic regression (LGR). In the non-DDI group, the area under ROC curve (AUC) of RF (0.912) was excellent, followed by that of MLP (0.813), CART (0.791), and C4.5 (0.784); the remaining classifiers performed poorly. For the DDI group, the AUC of RF (0.892) was the best, followed by CART (0.795), MLP (0.777), and C4.5 (0.774); the other classifiers' performances were less than ideal. The decision tree-based approaches and MLP exhibited markedly superior accuracy performance, regardless of DDI status. Although digoxin is a high-alert medication, its initial dose can be accurately determined by using data mining techniques such as decision tree-based and MLP approaches. Developing a dosage decision support system may serve as a supplementary tool for clinicians and also increase drug safety in clinical practice.
\end{abstract}

\section{Introduction}

Digoxin is the only oral heart medication approved by the United States Food and Drug Administration for use in enhancing positive inotropic effects and treating congestive heart failure (CHF). Because of its narrow therapeutic range and high drug-drug interactions (DDIs), digoxin is on the list of high-alert medications. Concentration changes of digoxin in the body are related to factors such as physiological characteristics, disease state, and coadministered drugs. Inappropriate dosages resulting in excessive drug concentrations in the body can cause numerous adverse reactions that affect the functioning of multiple organs $[1,2]$.

Digoxin toxicity is ranked fourth among adverse drug events (ADEs) involving older adults [3]. Digoxin also ranks among the top ten drugs requiring the interdisciplinary approaches of physicians, pharmacists, and nurses to jointly provide drug-related care [4]. Although the digoxin usage rate declined from $31.4 \%$ in 2001 to $23.5 \%$ in 2004 , this trend was not reflected in the number of hospitalizations for 
digoxin toxicity, which indicates the difficulty involved in determining the appropriate dosage [5].

Digoxin-specific antibodies can be acutely administered to patients with digoxin toxicity; however, because adverse reactions occur directly in the heart or in the central nervous system, death may occur within a short time in severe cases. A previous study reported that when the serum digoxin concentration (SDC) exceeds $1.2 \mathrm{ng} / \mathrm{mL}$, the mortality rate of patients who administered digoxin was $11.8 \%$ higher than that of patients in the placebo group [6].

Digoxin toxicity elevates mortality rates and increases medical costs. Gandhi et al. [7] indicated that the mean length of stay in the hospital as a result of digoxin toxicity was $3.3 \pm 1.2$ days, and the mean overall cost associated with digoxin toxicity was US\$4087.05 \pm US\$2659.76. Moreover, these increased expenditures correlated significantly with increased SDC. According to reports in $[1,8]$, approximately $50 \%$ of digoxin toxicity cases are preventable, which motivated us to improve the treatment outcomes of digoxin, reduce the incidence rate of digoxin toxicity, and minimize the related medical costs.

Recent studies [9-17] on pharmacogenetics have investigated the influence of the ABCB1 gene polymorphism on SDC; however, the correlation between these two factors remains unclear, and conclusions have been inconsistent. Hoffmeyer et al. [11] reported an association of the ABCB1 gene polymorphism with SDC, and some investigators $[12,13,16]$ confirmed the result of Hoffmeyer et al. [11]; on the other hand, Sakaeda et al. [15] contended that ABCB1 genotypes minimally influences digoxin pharmacokinetics, and Kurzawski et al. [17] noted that compared with other factors (i.e., age, diseases, herbs, and coadministered drugs), $\mathrm{ABCB} 1$ polymorphism slightly influences the digoxin level. In clinical practice, genetic testing is expensive and timeconsuming, and its practical benefits in terms of determining an appropriate dosage remain unconfirmed. Therefore, using relatively inexpensive and accessible laboratory data to construct multiple prediction models is a feasible direction for future studies.

Recently, many researchers have applied statistical-based techniques to construct digoxin dosage prediction equations or models from clinical features. However, those studies considered relatively few variables and used tools that possessed poor prediction ability. Therefore, how to develop a robust digoxin dosage prediction model from clinical records is still a challenging task.

This study collaborated with a medical center in Taiwan and collected personal information of 307 inpatients who received digoxin treatment during the period of 2004-2013. Numerous studies have proven that machine learning techniques demonstrate superior performance on building prediction models. Therefore, a number of machine learning techniques, including decision tree (C4.5), $k$-nearest neighbors $(\mathrm{kNN})$, classification and regression tree (CART), randomForest (RF), multilayer perceptron (MLP), and logistic regression (LGR), were used to construct prediction models for digoxin dosage adequacy. To evaluate the performance of the constructed classification systems, the accuracy, sensitivity, specificity, and the area under the ROC curve (AUC) of each classifier were assessed.

The paper is structured as follows: Section 2 presents the work related to digoxin dosage determination. Section 3 provides the preparation of data, experimental setup, and performance measures. Sections 4 and 5 present thorough experimental results and discussions. Section 6 concludes our study.

\section{Related Work}

In recent years, a number of methods have been proposed to predict digoxin dosage and treatment appropriateness (as shown in Table 1). These methods are largely statisticalbased techniques, such as regression model, Konishi equation, and the pharmacokinetics-based nonlinear mixedeffect modeling (NONMEM). For example, the NONMEM is a widely implemented method that enables physicians to estimate the appropriate dosage of a drug by calculating the SDC or clearance rate. When establishing a pharmacokinetics model, one must possess a complete understanding of various statistical models, including parameters related to pharmacokinetics structural models. However, Tolle et al. [37] argued that the performance of pharmacokinetic modeling can be significantly affected by population characteristics of patients and many other interference factors. In addition, the pharmacological properties of digoxin are more complex than those of other drugs, and its parameters are not easily established. As a result, developing a statisticalbased model to digoxin dosage prediction is highly difficult.

Few recent studies have applied machine learning techniques to digoxin treatment decisions. For example, Albert et al. [8] applied artificial neural network (ANN) to predict toxicity after digoxin administration, and results showed the performance of the neural network model better than the logistic regressions one in both sensitivity and specificity. Martín et al. [24] also adopted ANN to predict digoxin toxicity and reported similar outcomes. However, ANN-based methods have poor interpretability. Clinicians cannot easily understand the output generated by ANN, which limit its clinical applications.

Previous studies reveal that considering SDC or the clearance of digoxin (CL) provides promising prediction results. In clinical practice, physicians normally test patients' SDC, and adjust medication dose according to therapeutic drug monitoring (TDM) and personal experience. However, for patients receiving such medications for the first time, physicians are unable to perform dose evaluation via SDC or CL. Therefore, our primary goal of this study was to apply machine learning techniques to propose an alternative approach that could facilitate the prediction of the initial dosage adequacy of digoxin.

In addition, previous studies utilized different population of ethnic groups to develop various prediction models, which reveals that ethnicity is an important factor in digoxin dosing. To the best of our knowledge, this study first adopts machine learning techniques to construct digoxin dosage prediction models for the Chinese ethnic group; our 


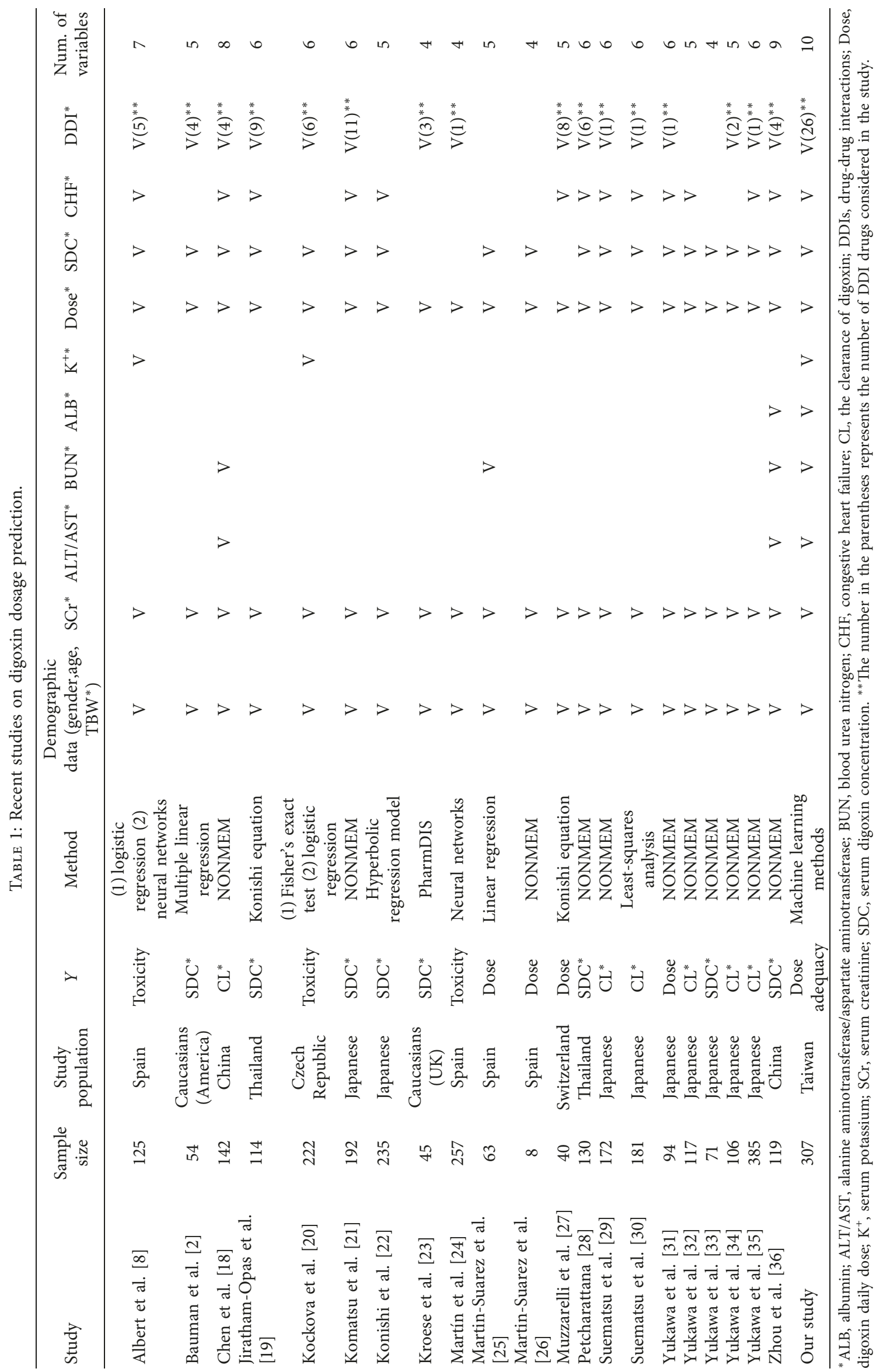


results can provide significant information in clinical decision support.

Our main contribution of this work is to construct more practical and robust prediction systems, including all drugs having DDI when combined with digoxin is indispensable. The research results indicated that using a high-performance classification model can effectively improve the prediction accuracy rate, thereby confirming the value of this technique in clinical applications.

\section{Materials and Methods}

3.1. Data. Research data were obtained through a medical records database at a medical center in Taiwan; specifically, the medical records were of patients who had been hospitalized and had their conditions treated with digoxin between 2004 and 2013. In addition, because some of the patients may have taken digoxin prior to hospitalization, a 3-month washout period was imposed to ensure the accuracy of the prediction models after considering the drug's half-life (i.e., 36 to 48 hours with normal renal function) [38]. Therefore, patients who had consumed digoxin within 3 months prior to hospitalization were excluded from this study. The Chang Gung Memorial Hospital Institutional Review Board approved the study protocol (105-0542C). Written consent from the study was deemed unnecessary because the dataset comprises only deidentified secondary data for research purposes, and the Chang Gung Memorial Hospital Institutional Review Board issued a formal written waiver of the need for consent and approved the study.

In addition, the medical records indicated that most of the patients had been prescribed half a digoxin pill per day (i.e., $0.125 \mathrm{mg}$ daily) as an initial dosage. To obtain more reliable results, therefore, this study only selected the patients who had been prescribed $0.125 \mathrm{mg}$ daily as the study samples.

To construct the prediction models, each patient was classified based on whether the treatment was considered to be appropriate or inappropriate (i.e., dependent variable). Currently, SDC is the primary reference indicator for determining the appropriate dosage of digoxin; if the SDC is controlled at $0.5-0.9 \mathrm{ng} / \mathrm{mL}$, this can effectively reduce allcause mortality and hospitalization rates $[2,20]$. Therefore, a patient with SDC being controlled at $0.5-0.9 \mathrm{ng} / \mathrm{mL}$ after his/her initial digoxin dosage was defined as having an appropriate use of digoxin; otherwise, a patient was defined as having an inappropriate use of digoxin.

Each hospitalization record contained 10 independent variables, including demographic information (i.e., sex, age, and weight) and test data (i.e., alanine aminotransferase (ALT), aspartate aminotransferase (AST), serum creatinine concentrations (SCr), blood urea nitrogen (BUN), albumin (ALB), and serum $\left(\mathrm{K}^{+}\right)$). Whether the patients had any specific illnesses (CHF) was also noted.

Furthermore, DDI was another critical factor on digoxin dosage determination and required consideration before constructing the prediction models because it can affect SDC performance and threaten the patient's life in severe cases
TABLE 2: A list of medicines causing major DDI when combined with digoxin.

\begin{tabular}{lccc}
\hline Amiodarone & Dronedarone & Norepinephrine & Spironolactone \\
Alprazolam & Epinephrine & Oxytetracycline & Succinylcholine \\
Boceprevir & Erythromycin & Propafenone & Tetracycline \\
Calcium & Indomethacin & Propantheline & Thiazide \\
carbonate & diuretics \\
Clarithromycin & Itraconazole & Quinidine & Verapamil \\
Dopamine & Mifepristone & Ritonavir & \\
Doxycycline & Minocycline & Saquinavir & \\
\hline
\end{tabular}

$[1,2]$. Therefore, we referenced DDI information released by the Ministry of Health and Welfare in Taiwan and compared this information with records from the drug registry used at the studied medical center, subsequently identifying 26 drugs that are known to produce major DDIs when coadministered with digoxin (Table 2). The risk of DDI was considered for the patients who were prescribed any of the drugs listed in Table 2 while undergoing digoxin therapy, and the data observations were accordingly categorized as "with DDIs" or "without DDIs" before conducting the analysis.

To construct highly reliable digoxin dosage prediction models, the collected 307 clinical cases were then further divided into two datasets: one containing 222 cases with DDIs (i.e., DDI group) and the other containing 85 cases without DDI (i.e., non-DDI group).

3.2. Experimental Setup. To develop prediction models for evaluating the appropriateness of initial dosage of digoxin, this study adopted Weka 3.7.3 (www.cs.waikato.ac. $\mathrm{nz} / \mathrm{ml} /$ weka), an open source machine learning software. A number of machine learning techniques were considered, including decision tree (C4.5), $k$-nearest neighbors $(\mathrm{kNN})$, classification and regression tree (CART), randomForest (RF), multilayer perceptron (MLP), and logistic regression (LGR). In addition, the prediction performance of machine learning techniques can be significantly influenced by the internal parameter setting. To optimize the prediction performance of the selected techniques, the CVParameterSelection metalearner module implemented in Weka was used. In this module, we first selected a prediction technique and specified various parameter combinations. The algorithm then automatically searched the optimal parameter setting based on the best prediction results using cross validation. The parameter settings used in this study is listed in Table 3.

Previous study showed that the class imbalance problem can significantly affect the learning performance [39]. To improve the classification performance, a resample module in Weka is adopted to modify the distribution of instances of two classes. Specifically, the distribution of the class label is modified to be almost identical by oversampling the inadequate class and undersampling the adequate class. In addition, the random resample technique is applied thirty times to construct datasets; for each generated dataset, tenfold cross validation is then applied in all the experimental evaluations [40]. Specifically, each dataset is partitioned into 
Table 3: Parameter settings in WEKA.

\begin{tabular}{|c|c|c|c|}
\hline Method & Parameters & Value/Range & Best parameter setting \\
\hline \multirow{2}{*}{$\mathrm{J} 48$} & Confidence factor & $0.1-0.5$ & 0.25 \\
\hline & Minimum number of instances per leaf & $2-50$ & 2 \\
\hline IBk & Number of neighbors & $2-10$ & 2 \\
\hline SimpleCART & Minimum number of instances per leaf & $2-50$ & 2 \\
\hline \multirow{2}{*}{ RandomForest } & Number of trees & $5-10$ & 10 \\
\hline & Number of attributes to be used in random selection & $2-8$ & 4 \\
\hline \multirow{4}{*}{ Multilayer perceptron } & Number of hidden nodes & $3-14$ & 7 \\
\hline & Learning rate & $0.1-0.6$ & 0.3 \\
\hline & Momentum factor & $0-0.9$ & 0.2 \\
\hline & Maximum number of epochs & $300-1000$ & 500 \\
\hline \multirow{2}{*}{ AdaBoostM1 } & Number of iterations & 10 & 10 \\
\hline & Weight threshold for pruning & 100 & 100 \\
\hline
\end{tabular}

TABle 4: Confusion matrix.

\begin{tabular}{cccc}
\hline & & \multicolumn{2}{c}{ Predicted class } \\
& & Adequate & Inadequate \\
\hline \multirow{2}{*}{ Actual class } & Adequate & $a$ & $b$ \\
& Inadequate & $c$ & $d$ \\
\hline
\end{tabular}

ten complementary subsets, wherein any nine were used for model training, and the remaining subset was used for model testing.

3.3. Performance Measures. To evaluate the performance of the constructed classification systems (i.e., prediction models), the accuracy, sensitivity, and specificity of each classifier was assessed. These were measured using a confusion matrix, as shown in Table 4.

The prediction accuracy, sensitivity, and specificity were obtained using the following formulas:

$$
\begin{aligned}
\text { Accuracy } & =\frac{a+d}{a+b+c+d}, \\
\text { Sensitivity } & =\frac{a}{a+b}, \\
\text { Specificity } & =\frac{d}{c+d} .
\end{aligned}
$$

In addition to use the performance measures, the receiver operating characteristic (ROC) curve is plotted, and the area under the ROC curve (AUC) is also calculated. The ROC curve can illustrate the performance of a binary classifier as its discrimination threshold is varied. It can be generated by plotting the sensitivity against (1-specificity) at different discrimination threshold settings. When using normalized units, the AUC is equal to the probability that a classifier will rank a randomly chosen positive (i.e., adequate digoxin dosage) sample higher than a randomly chosen negative (i.e., inadequate digoxin dosage) one. Hosmer and Lemeshow [41] provide general rules to categorize the evaluation performance using the AUC as "excellent" if AUC $\geq 0.9$, "good" if $0.9>A U C \geq 0.8$, "fair" if $0.8>$ AUC $\geq 0.7$, "poor" if $0.7>A U C \geq 0.6$, and "very poor" if $\mathrm{AUC}<0.6$.

\section{Results}

Table 5 lists variables and descriptive statistics of the non-DDI and DDI groups. As mentioned earlier, there were 222 inpatient cases drawn from the group with DDIs and 85 cases without DDIs, resulting in 307 valid clinical cases in total.

Table 6 shows the experimental results of the non-DDI and DDI groups, which were analyzed using 6 specific classification techniques. Sensitivity, specificity, accuracy, and area under the curve (AUC) were employed to evaluate the effectiveness of the prediction models. To facilitate result interpretation, the means and standard deviations of the above four metrics from 30 datasets are listed in the table.

Regarding the accuracy of the prediction models for the non-DDI group, the RF result was the most favorable (83.9\%), followed by the MLP result (80.9\%), and CART and C4.5 yielded accuracy rates above $75 \%$. The remaining classifiers performed poorly, all of which yielded accuracy rates below $65 \%$. Because of its complex medication regimens, the DDI group generally exhibited prediction accuracies that were lower than those of the non-DDI group. However, for the DDI group, the RF (80.5\%) continued to yield the most favorable result $(80.5 \%)$, followed by the MLP, CART, and C4.5. The other classifiers performed poorly, yielding no result higher than $60.2 \%$. Overall, the decision tree-based approaches (i.e., RF, C4.5, and CART) and MLP exhibited markedly superior accuracy performance, regardless of DDI status.

The AUC performance values were distributed in the 0.533-0.912 range. In the non-DDI group, RF was excellent, followed by MLP, CART and C4.5; the remaining classifiers performed poorly. For the DDI group, the RF performance was the best (0.892; good), followed by CART, MLP, and C4.5; the other classifiers' performances were less than ideal.

Finally, a comprehensive assessment of the various indicators revealed that, regardless of DDI status, the decision tree-based classifiers clearly outperformed the $\mathrm{kNN}$ and LGR classifiers, demonstrating the superior accuracy of the decision tree-based approaches for predicting appropriate dosages.

\section{Discussion}

The safety of using high-alert medications such as digoxin is a pressing topic $[1,2,8]$. Previous studies have employed 
TABLE 5: Summary statistics for the non-DDI and DDI groups.

\begin{tabular}{|c|c|c|c|c|}
\hline \multirow{2}{*}{ Variable } & \multicolumn{2}{|c|}{ Non-DDI group } & \multicolumn{2}{|c|}{ DDI group } \\
\hline & Range & Summary statistics & Range & Summary statistics \\
\hline Gender & Male/female & $\begin{array}{c}\text { Male: } 40 \\
\text { Female: } 45\end{array}$ & Male/female & $\begin{array}{c}\text { Male: } 121 \\
\text { Female: } 101\end{array}$ \\
\hline Age (years) & 38 to 94 & $\mu=73.95, \sigma=10.53$ & 23 to 101 & $\mu=74.84, \sigma=11.28$ \\
\hline Weight (kg) & 35 to 89 & $\mu=57.37, \sigma=10.62$ & 33 to 105 & $\mu=55.95, \sigma=12.90$ \\
\hline SDC & 0.2 to 2.4 & $\mu=0.628, \sigma=0.470$ & 0.2 to 4.3 & $\mu=0.85, \sigma=0.59$ \\
\hline ALT & 8 to 475 & $\mu=46.05, \sigma=81.74$ & 5 to 1381 & $\mu=85.26, \sigma=191.95$ \\
\hline AST & 17 to 1776 & $\mu=169.69, \sigma=437.21$ & 12 to 2615 & $\mu=105.77, \sigma=290.97$ \\
\hline $\mathrm{SCr}$ & 0.38 to 3.55 & $\mu=1.046, \sigma=0.653$ & 0.29 to 12.1 & $\mu=1.361, \sigma=1.270$ \\
\hline BUN & 1.9 to 83 & $\mu=21.43, \sigma=14.50$ & 2 to 219 & $\mu=35.49, \sigma=30.10$ \\
\hline ALB & 1.4 to 4.2 & $\mu=2.913, \sigma=0.597$ & 1.1 to 4.2 & $\mu=2.546, \sigma=0.611$ \\
\hline $\mathrm{K}+$ & 2.5 to 5.4 & $\mu=3.96, \sigma=0.614$ & 2.69 to 6.80 & $\mu=3.979, \sigma=0.723$ \\
\hline $\mathrm{CHF}$ & Yes/no & Yes: 35/no: 50 & Yes/no & Yes: 132/no: 90 \\
\hline
\end{tabular}

TABle 6: Performance evaluation of the classifiers for the non-DDI and DDI groups.

\begin{tabular}{|c|c|c|c|c|c|}
\hline Group & Method & Sensitivity & Specificity & Accuracy & AUC \\
\hline \multirow{6}{*}{ Non-DDI } & C4.5 & $0.705 / 0.091$ & $0.806 / 0.078$ & $0.759 / 0.061$ & $0.784 / 0.065$ \\
\hline & CART & $0.696 / 0.095$ & $0.825 / 0.067$ & $0.765 / 0.055$ & $0.791 / 0.057$ \\
\hline & RF & $0.782 / 0.090$ & $0.888 / 0.054$ & $00.839 / 0.041$ & $0.912 / 0.032$ \\
\hline & $\mathrm{kNN}$ & $0.619 / 0.172$ & $0.547 / 0.162$ & $0.592 / 0.068$ & $0.606 / 0.070$ \\
\hline & LGR & $0.566 / 0.145$ & $0.715 / 0.117$ & $0.648 / 0.078$ & $0.661 / 0.097$ \\
\hline & MLP & $0.741 / 0.091$ & $0.871 / 0.057$ & $0.809 / 0.059$ & $0.813 / 0.071$ \\
\hline \multirow{6}{*}{ DDI } & $\mathrm{C} 4.5$ & $0.701 / 0.060$ & $0.759 / 0.050$ & $0.732 / 0.029$ & $0.774 / 0.030$ \\
\hline & CART & $0.728 / 0.051$ & $0.776 / 0.050$ & $0.754 / 0.031$ & $0.795 / 0.031$ \\
\hline & $\mathrm{RF}$ & $0.790 / 0.050$ & $0.817 / 0.043$ & $0.805 / 0.027$ & $0.892 / 0.020$ \\
\hline & $\mathrm{kNN}$ & $0.545 / 0.094$ & $0.651 / 0.087$ & $0.602 / 0.042$ & $0.634 / 0.048$ \\
\hline & LGR & $0.464 / 0.139$ & $0.621 / 0.145$ & $0.551 / 0.042$ & $0.556 / 0.058$ \\
\hline & MLP & $0.745 / 0.058$ & $0.799 / 0.042$ & $0.774 / 0.037$ & $0.777 / 0.051$ \\
\hline
\end{tabular}

statistical models $[1,2,20,22,25]$ and pharmacokinetics $[18,21,23,26,28,29,31-36]$, and data mining and machine learning techniques have only recently been adopted to improve model predictability $[8,24]$. This study investigated decision tree-based approaches, which were identified to exhibit an average performance superior to that of other techniques. In addition, the information obtained through decision tree-based approaches can be presented as if-then rules that physicians can refer to determine the appropriate dosage of digoxin to prescribe to patients.

Among the investigated three decision tree-based approaches, an RF classifier exhibited the optimal prediction performance. $\mathrm{RF}$ is an ensemble classifier that combines bagging and decision tree techniques. Let $m$ be the number of variables and $n$ be the number of instances, the time complexity for constructing an unpruned decision tree is $O(m \cdot n \cdot \log (n))$. In building RF classifier, one should define the number of bootstrap sample sets (denoted as nbt) and the number of variables that can be randomly selected for each sample set (denoted as nvar). Therefore, the complexity of building an RF classifier is $O(n b t \cdot \operatorname{nvar} \cdot n \cdot \log (n))$.

In addition to comparing the predictive capabilities of various classification techniques, this study further evaluated the importance of multiple variables to provide a reference for clinicians. As shown in Table 5, 10 variables were used in analysis, which is more than the number of variables examined in previous studies. Furthermore, previous studies have evaluated only 8 drug types for DDIs; in this study, a total of 26 drugs with major DDI effects were included.

By calculating gain ratios, we found that the most crucial variables influencing dosage appropriateness were in the order SCr, serum $\mathrm{K}^{+}, \mathrm{CHF}$, and DDI (as shown in Table 7). Following a discussion with the physicians and pharmacists at the case medical center, the validity of the aforementioned results is described as follows.

First, SCr is a major indicator of renal function. Because digoxin in the body is mainly excreted through the kidneys, poor renal function may result in a longer half-life of digoxin in the body and an elevated SDC, thereby inducing digoxin toxicity. We presented that renal function plays a significant role for the SDC, and the finding is consistent with the results of earlier studies about digoxin doses in patients with renal failure [42-45]. Therefore, clinicians should consider prescribing a low dosage of digoxin to patients with poor renal function. Furthermore, our study indicated that serum $\mathrm{K}^{+}$is also a critical factor of dosage appropriateness. Digoxin's primary mechanism of action involves inhibition of the sodium potassium adenosine triphosphatase $(\mathrm{Na}+/ \mathrm{K}+$ ATPase), mainly in the myocardium. Because potassium and digoxin compete for the same ATPase-binding site, excessively low concentrations of potassium ions in the body cause the cardiomyocytes to absorb additional digoxin, thereby increasing toxicity risk [46-50]. Therefore, clinicians should monitor their patients' electrolyte levels (particularly 
TABLE 7: Ranking of selected variables by gain ratio.

\begin{tabular}{lcc}
\hline Rank & Variable & Gain ratios \\
\hline 1 & SCr & 0.0826 \\
2 & Serum K $K^{+}$ & 0.0428 \\
3 & CHF & 0.0129 \\
4 & DDI & 0.0101 \\
\hline
\end{tabular}

serum $\mathrm{K}^{+}$) to facilitate dosage adjustment. Second, one of the major indications for using digoxin is CHF, and accumulative evidences indicate that low-dose digoxin can reduce hospitalization and mortality in patients with heart failure [51-53]. However, a history of CHF may influence the metabolism of digoxin. Previous studies showed that digoxin absorption is slower, and peak concentration is lower in patients with CHF than in healthy volunteers due to reduced gastrointestinal motility, congestion in the gut wall, and reduced splanchnic blood flow in patients with CHF [5456], thereby causing changes of SDC. Finally, DDI involving high-alert medications has gained considerable attention in the field of medicine, particularly digoxin, which has a narrow therapeutic concentration range $[2,8,18,20,23$, $24,27-31,34-36]$. DDI involving digoxin can easily lead to an elevated SDC and subsequent toxicity reaction [57]. Although this study included only drugs that are known to produce major DDI with digoxin, the results are sufficient for confirming the importance of DDI. The results also confirmed that adequate laboratory information can effectively assist physicians in determining the appropriate dosage.

\section{Conclusion}

Medication safety has received considerable attention in the medical community in recent years. It is particularly crucial for high-alert medications that have a narrow therapeutic range or easily induce toxicity. Digoxin is a high-alert medication, and prescribing an inappropriate dosage of digoxin can easily cause severe side effects and even fatality. The objective of this study was to predict the appropriateness of the initial dosage of digoxin. Six classification techniques were adopted to establish multiple classification models for predicting dosage appropriateness. The medical records of 307 hospitalized patients were used to confirm that the prediction accuracy rates of all adopted techniques exceeded those of the physicians in the actual patient cases. Overall, the RF prediction model exhibited the optimal effectiveness; the decision tree-based approaches exhibited favorable performance and can be used by clinicians as aid for making clinical dosage decisions.

Although digoxin is characterized by complex pharmacological properties, this study confirmed that the adequate use of laboratory data and consideration of numerous variables can yield favorable prediction effectiveness. In conjunction with clinical experience, the suggested prediction models can facilitate clinicians making a proper decision in practice. The improvement in the safe use of digoxin will be of benefit to both clinicians and patients.

Some limitations of the present study should be addressed because they may restrict generalizability and are indicative of the need for further research. First, the data used in this study were collected from a single medical institution. Proceeding with the evaluations of clinical cases from other hospitals is critical for confirming the validity of the model. Second, other potentially valuable features, such as data collected from the nursing information system and clinical pathway, can be considered for use in the model. Finally, because most of the inpatients took half a digoxin pill per day as an initial dosage in the case hospital, this study only focused on evaluating the adequacy of prescribing $0.125 \mathrm{mg}$ digoxin as an initial dosage for the inpatients daily. Future studies may directly predict adequate digoxin dosage when a large enough number of samples are collected.

\section{Data Availability}

The data used to support the findings of this study are available from the corresponding author upon request.

\section{Conflicts of Interest}

The authors declare that they have no conflicts of interest.

\section{Authors' Contributions}

Ya-Han $\mathrm{Hu}$ and Chun-Tien Tai contributed equally to this work.

\section{Acknowledgments}

This research was supported in part by the Chang Gung Memorial Hospital (Grant no. CMRPG6F0201) and the Center for Innovative Research on Aging Society (CIRAS) from The Featured Areas Research Center Program within the framework of the Higher Education Sprout Project by Ministry of Education (MOE) in Taiwan.

\section{References}

[1] J. L. Bauman, R. J. DiDomenico, and W. L. Galanter, "Mechanisms, manifestations, and management of digoxin toxicity in the modern era," American Journal of Cardiovascular Drugs, vol. 6, no. 2, pp. 77-86, 2006.

[2] J. L. Bauman, R. J. DiDomenico, M. Viana, and M. Fitch, “A method of determining the dose of digoxin for heart failure in the modern era," Archives of Internal Medicine, vol. 166, no. 22, pp. 2539-2545, 2006.

[3] J. H. Gurwitz, T. S. Field, J. Avorn et al., "Incidence and preventability of adverse drug events in nursing homes," American Journal of Medicine, vol. 109, no. 2, pp. 87-94, 2000.

[4] N. P. Markota, I. Markota, M. Tomic, and A. Zelenika, "Inappropriate drug dosage adjustments in patients with renal impairment," Journal of Nephrology, vol. 22, no. 4, pp. 497501, 2009.

[5] Z. Hussain, J. Swindle, and P. J. Hauptman, "Digoxin use and digoxin toxicity in the post-DIG trial era," Journal of Cardiac Failure, vol. 12, no. 5, pp. 343-346, 2006.

[6] S. S. Rathore, J. P. Curtis, Y. Wang, M. R. Bristow, and H. M. Krumholz, "Association of serum digoxin concentration and outcomes in patients with heart failure," JAMA, vol. 289, no. 7, pp. 871-878, 2003. 
[7] A. J. Gandhi, P. H. Vlasses, D. J. Morton, and J. L. Bauman, "Economic impact of digoxin toxicity," Pharmacoeconomics, vol. 12, no. 2, pp. 175-181, 1997.

[8] A. Albert, A. J. Serrano, E. Soria, and N. V. Jiménez, "Clinical decision support system to prevent toxicity in patients treated with digoxin," in Intelligent Medical Technologies and Biomedical Engineering: Tools and Applications, pp. 1-21, Medical Information Science Reference, Hershey, PA, USA, 2010.

[9] L. Becquemont, C. Verstuyft, R. Kerb et al., "Effect of grapefruit juice on digoxin pharmacokinetics in humans," Clinical Pharmacology and Therapeutics, vol. 70, no. 4, pp. 311-316, 2001.

[10] T. Gerloff, M. Schaefer, A. Johne et al., "MDR1 genotypes do not influence the absorption of a single oral dose of $1 \mathrm{mg}$ digoxin in healthy white males," British Journal of Clinical Pharmacology, vol. 54, no. 6, pp. 610-616, 2002.

[11] S. Hoffmeyer, O. Burk, O. Von Richter et al., "Functional polymorphisms of the human multidrug-resistance gene: multiple sequence variations and correlation of one allele with P-glycoprotein expression and activity in vivo," Proceedings of the National Academy of Sciences, vol. 97, no. 7, pp. 3473$3478,2000$.

[12] A. Johne, K. Kopke, T. Gerloff et al., "Modulation of steadystate kinetics of digoxin by haplotypes of the P-glycoprotein MDR1 gene," Clinical Pharmacology and Therapeutics, vol. 72, no. 5, pp. 584-594, 2002.

[13] Y. Kurata, I. Ieiri, M. Kimura et al., "Role of human MDR1 gene polymorphism in bioavailability and interaction of digoxin, a substrate of P-glycoprotein," Clinical Pharmacology and Therapeutics, vol. 72, no. 2, pp. 209-219, 2002.

[14] T. Sakaeda, T. Nakamura, M. Horinouchi et al., "MDR1 genotyperelated pharmacokinetics of digoxin after single oral administration in healthy Japanese subjects," Pharmaceutical Research, vol. 18, no. 10, pp. 1400-1404, 2001.

[15] T. Sakaeda, "MDR1 genotype-related pharmacokinetics: fact or fiction?," Drug Metabolism and Pharmacokinetics, vol. 20, no. 6, pp. 391-414, 2005.

[16] C. Verstuyft, M. Schwab, E. Schaeffeler et al., "Digoxin pharmacokinetics and MDR1 genetic polymorphisms," European Journal of Clinical Pharmacology, vol. 58, no. 12, pp. 809-812, 2003.

[17] M. Kurzawski, L. Bartnicka, M. Florczak, W. Gomik, and M. Drozdzik, "Impact of ABCB1 (MDR1) gene polymorphism and P-glycoprotein inhibitors on digoxin serum concentration in congestive heart failure patients," Pharmacological Reports, vol. 59, no. 1, pp. 107-111, 2007.

[18] R. Chen, S. L. Zou, M. L. Wang et al., "Population pharmacokinetics of digoxin in elderly patients," European Journal of Drug Metabolism and Pharmacokinetics, vol. 38, no. 2, pp. 115-121, 2013.

[19] J. Jiratham-Opas, R. Kanjanavanit, W. Wongcharoen et al., "Can available mathematical models predict serum digoxin levels in Thai patients?," Journal of Clinical Pharmacy and Therapeutics, vol. 43, no. 3, pp. 377-384, 2018.

[20] R. Kockova, J. Skvaril, M. Cernohous, M. Maly, V. Kocka, and A. Linhart, "Five year two center retrospective analysis of patients with toxic digoxin serum concentration," International Journal of Cardiology, vol. 146, no. 3, pp. 447-448, 2011.

[21] T. Komatsu, M. Morita, F. Miyaji, T. Inomata, J. Ako, and K. Atsuda, "Population pharmacokinetics and optimization of the dosing regimen of digoxin in adult patients," Journal of Pharmaceutical Health Care and Sciences, vol. 1, no. 1, p. 25, 2015.

[22] H. Konishi, S. Shimizu, M. Chiba, T. Minouchi, M. Koida, and A. Yamaji, "Predictive performance of serum digoxin concentration in patients with congestive heart failure by a hyperbolic model based on creatinine clearance," Journal of Clinical Pharmacy and Therapeutics, vol. 27, no. 4, pp. 257-265, 2002.

[23] W. L. G. Kroese, A. J. Avery, B. S. P. Savelyich et al., "Assessing the accuracy of a computerized decision support system for digoxin dosing in primary care: an observational study," Journal of Clinical Pharmacy and Therapeutics, vol. 30, no. 3, pp. 279-283, 2005.

[24] J. D. Martín, E. Soria, G. Camps, A. J. Serrano, J. R. Sepúlveda, and V. Jiménez, "Neural networks as effective techniques in clinical management of patients: some case studies," Transactions of the Institute of Measurement and Control, vol. 26, no. 3, pp. 169-183, 2004.

[25] A. Martin-Suarez, D. G. González, J. F. M. Núñez, R. A. Albajar, and M. V. C. Hernández, "A new method for individualized digoxin dosing in elderly patients," Drugs and Aging, vol. 33, no. 4, pp. 277-284, 2016.

[26] A. Martin-Suarez, J. G. Sanchez-Hernandez, F. MedinaBarajas et al., "Pharmacokinetics and dosing requirements of digoxin in pregnant women treated for fetal supraventricular tachycardia," Expert Review of Clinical Pharmacology, vol. 10, no. 8, pp. 911-917, 2017.

[27] S. Muzzarelli, H. Stricker, O. Pfister et al., "Individual dosage of digoxin in patients with heart failure," QJM: An International Journal of Medicine, vol. 104, no. 4, pp. 309-317, 2011.

[28] S. Petcharattana, "Population pharmacokinetics of digoxin in Thai pediatric patients," Journal of the Medical Association of Thailand, vol. 92, no. 10, pp. 1324-1335, 2009.

[29] F. Suematsu, E. Yukawa, M. Yukawa et al., "Population-based investigation of relative clearance of digoxin in Japanese neonates and infants by multiple-trough screen analysis," European Journal of Clinical Pharmacology, vol. 57, no. 1, pp. 19-24, 2001.

[30] F. Suematsu, M. Minemoto, E. Yukawa, and S. Higuchi, "Population analysis for the optimization of digoxin treatment in Japanese paediatric patients," Journal of Clinical Pharmacy and Therapeutics, vol. 24, no. 3, pp. 203-208, 1999.

[31] M. Yukawa, E. Yukawa, F. Suematsu et al., "Determination of digoxin clearance in Japanese elderly patients for optimization of drug therapy," Drugs and aging, vol. 28, no. 10, pp. 831-841, 2011.

[32] M. Yukawa, E. Yukawa, F. Suematsu et al., "Population pharmacokinetic investigation of digoxin in Japanese infants and young children," Journal of Clinical Pharmacology, vol. 51, no. 6, pp. 857-863, 2011.

[33] E. Yukawa, K. Akiyama, F. Suematsu, M. Yukawa, and M. Minemoto, "Population pharmacokinetic investigation of digoxin in Japanese neonates," Journal of Clinical Pharmacy and Therapeutics, vol. 32, no. 4, pp. 381-386, 2007.

[34] E. Yukawa, F. Suematu, M. Yukawa et al., "Population pharmacokinetics of digoxin in Japanese patients," Clinical Pharmacokinetics, vol. 40, no. 10, pp. 773-781, 2001.

[35] E. Yukawa, T. Honda, S. Ohdo, S. Higuchi, and T. Aoyama, "Population-based investigation of relative clearance of digoxin in Japanese patients by multiple trough screen analysis: an update," Journal of Clinical Pharmacology, vol. 37, no. 2, pp. 92-100, 1997.

[36] X. D. Zhou, Y. Gao, Z. Guan, Z. D. Li, and J. Li, "Population pharmacokinetic model of digoxin in older Chinese patients and its application in clinical practice," Acta Pharmacologica Sinica, vol. 31, no. 6, pp. 753-758, 2010.

[37] K. M. Tolle, H. Chen, and H. Chow, "Estimating drug/plasma concentration levels by applying neural networks to pharmacokinetic data sets," Decision Support Systems, vol. 30, no. 2, pp. 139-151, 2000. 
[38] M. Gheorghiade, K. F. Adams, and W. S. Colucci, "Digoxin in the management of cardiovascular disorders," Circulation, vol. 109, no. 24, pp. 2959-2964, 2004.

[39] P-N. Tan, M. Steinbach, and V. Kumar, Introduction to Data Mining, Pearson, Chennai, India, 2006.

[40] E. Kretschmann and R. Apweiler, "Automatic rule generation for protein annotation with the C4.5 data-mining algorithm applied on peptides in Ensembl," in Proceedings of in German Conference on Bioinformatics, pp. 53-57, Braunschweig, Germany, October 2001.

[41] D. W. Hosmer Jr., S. Lemeshow, and R. X. Sturdivant, "Applied logistic regression," in Wiley Series in Probability and Statistics, Vol. 398, John Wiley \& Sons, Hoboken, NJ, USA, 2013.

[42] N. Pawlosky, E. MacDonald, R. Patel, and S. Kanji, "Evaluation of digoxin concentration after loading dose in patients with renal dysfunction," Canadian Journal of Hospital Pharmacy, vol. 66, no. 2, p. 104, 2013.

[43] A. Bajraktarević, "Safety and efficacy of digoxin therapywhere are we now?," Journal of Pharmacy and Pharmacology, vol. 4, no. 3, pp. 128-134, 2016.

[44] E. E. Ohnhaus, H. R. Lenzinger, and R. L. Galeazzi, "Comparison of two different loading doses of digoxin in severe renal impairment," European Journal of Clinical Pharmacology, vol. 18, no. 6, pp. 467-472, 1980.

[45] L. L. Brunton, B. Chabner, and B. C. Knollmann, Goodman \& Gilman's the Pharmacological Basis of Therapeutics, McGrawHill Medical, New York, NY, USA, 2011.

[46] A. Bielecka-Dabrowa, D. P. Mikhailidis, L. Jones, J. Rysz, W. S. Aronow, and M. Banach, "The meaning of hypokalemia in heart failure," International Journal of Cardiology, vol. 158, no. 1, pp. 12-17, 2012.

[47] J. E. Macdonald and A. D. Struthers, "What is the optimal serum potassium level in cardiovascular patients?," Journal of the American College of Cardiology, vol. 43, no. 2, pp. 155-161, 2004.

[48] Z. D. Goldberger and A. L. Goldberger, "Therapeutic ranges of serum digoxin concentrations in patients with heart failure," American Journal of Cardiology, vol. 109, no. 12, pp. 1818$1821,2012$.

[49] K. E. Chan, J. M. Lazarus, and R. M. Hakim, "Digoxin associates with mortality in ESRD," Journal of the American Society of Nephrology, vol. 21, no. 9, 2010.

[50] T. W. Smith, E. M. Antman, P. L. Friedman, C. M. Blatt, and J. D. Marsh, "Part III digitalis glycosides: mechanisms and manifestations of toxicity," Progress in Cardiovascular Diseases, vol. 27, no. 1, pp. 21-56, 1984.

[51] A. Ahmed and F. Waagstein, "Low-dose digoxin and reduction in mortality and morbidity in heart failure," International Journal of Cardiology, vol. 136, no. 1, pp. 91-92, 2009.

[52] A. Ahmed, "Digoxin and reduction in mortality and hospitalization in geriatric heart failure: importance of low doses and low serum concentrations," Journals of Gerontology Series A: Biological Sciences and Medical Sciences, vol. 62, no. 3, pp. 323-329, 2007.

[53] D. J. van Veldhuisen, "Low-dose digoxin in patients with heart failure: Less toxic and at least as effective?," Journal of the American College of Cardiology, vol. 39, no. 6, pp. 954-956, 2002.

[54] G. C. Oliver, R. Tazman, and R. Frederickson, "Influence of congestive heart failure on digoxin level," in Proceedings of in Symposium on Digitalis, pp. 336-347, Oslo, Norway, February 1973.

[55] D. Berkowitz, M. N. Groll, and W. Likoff, "Malabsorption as a complication of congestive heart failure," American journal of cardiology, vol. 11, no. 1, pp. 43-47, 1963.
[56] D. L. Mann, D. P. Zipes, P. Libby, and R. O. Bonow, Braunwald's Heart Disease: A Textbook of Cardiovascular Medicine, Elsevier Health Sciences, Amsterdam, Netherlands, 2014.

[57] M. Vamos, J. W. Erath, and S. H. Hohnloser, "Digoxinassociated mortality: a systematic review and meta-analysis of the literature," European Heart Journal, vol. 36, no. 28, pp. 1831-1838, 2015. 


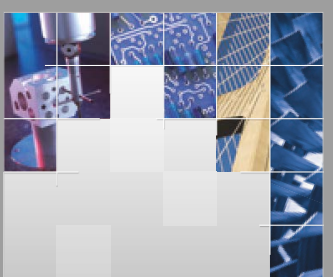

\section{Enfincering}
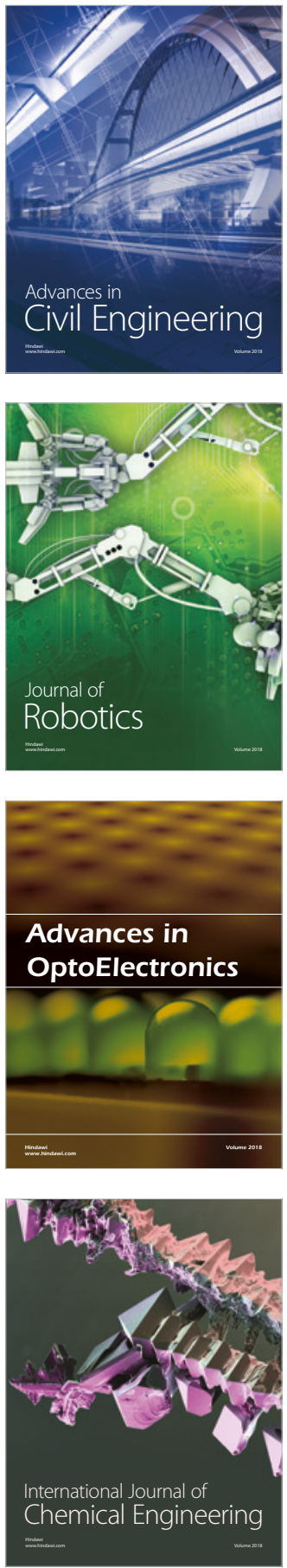

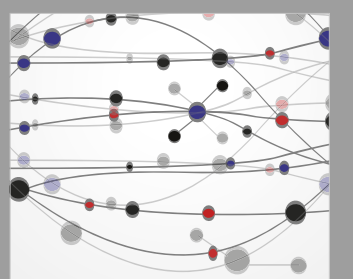

\section{Rotating \\ Machinery}

The Scientific World Journal

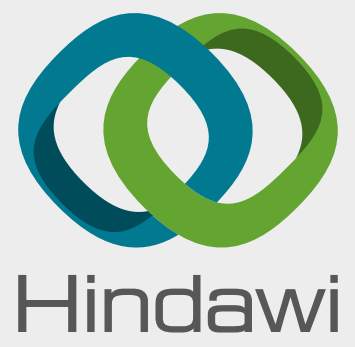

Submit your manuscripts at

www.hindawi.com
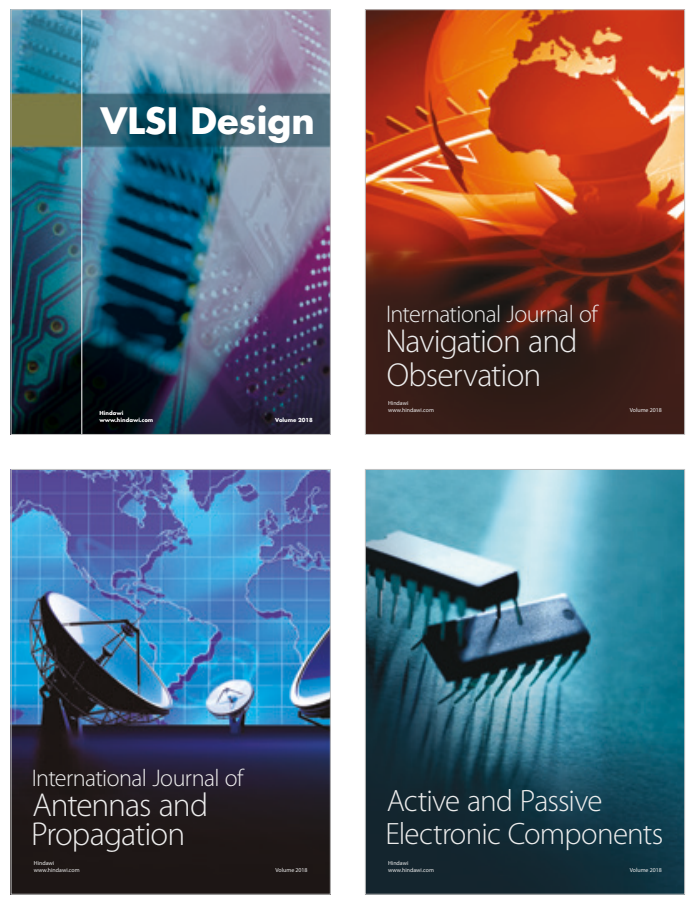
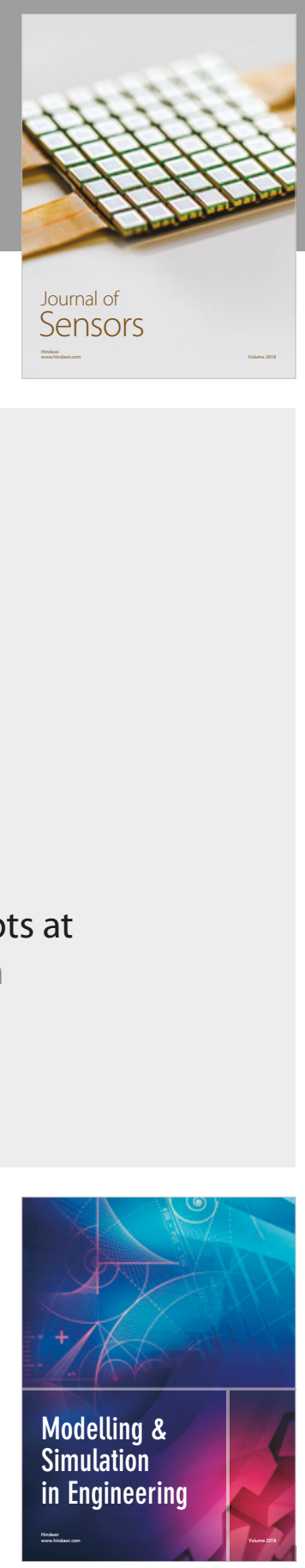

\section{Advances \\ Multimedia}
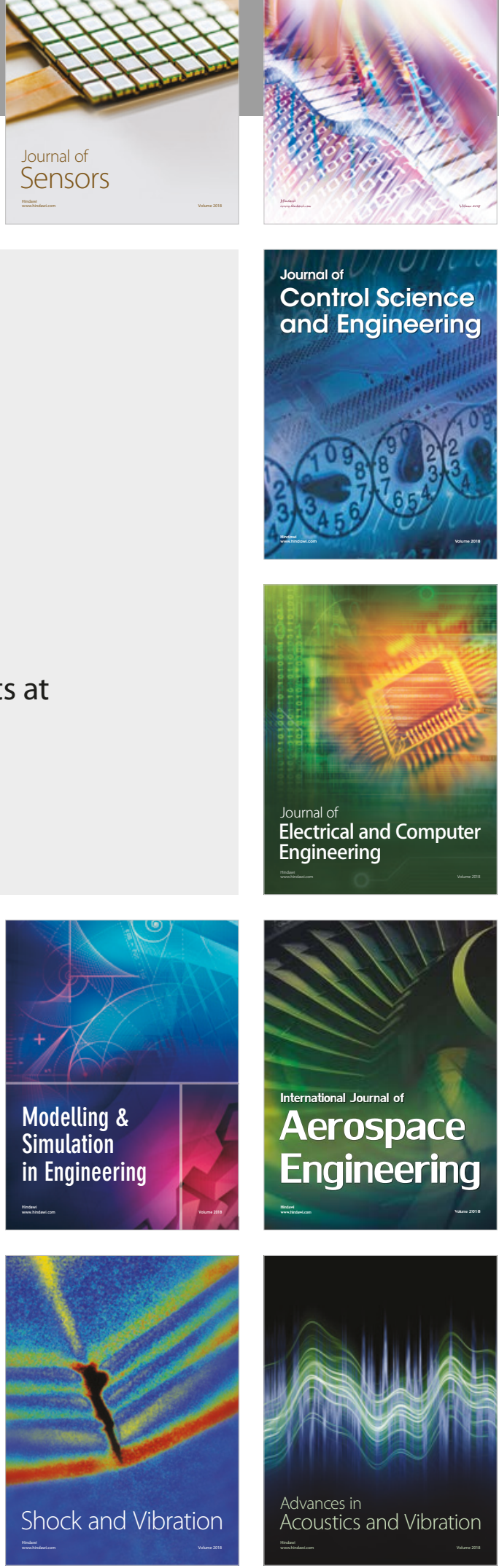